\title{
Prevalence of CTX-M beta-Lactamases in Escherichia coli from community-acquired urinary tract infections and associated risk factors among women in Cameroon
}

\author{
Ingrid Cécile Djuikoue*1,2 ${ }^{*}$ Omer Njajou ${ }^{1}$, Hortense Gonsu Kamga ${ }^{2}$, Charles Fokunang ${ }^{2}$, Adamo Bongoe ${ }^{1}$, Ela Ondo \\ Bruno $^{1}$, Paulin Tadjoung ${ }^{2}$, Abdou Aziz Linjouom², Caroline Kakam², Jeanne Ngogang ${ }^{1}$ \\ ${ }^{1}$ Unités de Santé Publique \& Epidémiologie, et de Microbiologie, Université des Montagnes, Bangangté, Cameroun \\ ${ }^{2}$ Département de Microbiologie et Maladies Infectieuses/Faculté de Médecine et des Sciences Biomédicales, Université de \\ Yaoundé, Yaoundé, Cameroun
}

Received: December 1, 2016

Accepted: January 6, 2017

Online Published: January 19, 2017

DOI: $10.5430 /$ jer.v3n1p51

URL: http://dx.doi.org/10.5430/jer.v3n1p51

\begin{abstract}
Background: In recent years, a worldwide dissemination of CTX-M beta-lactamase-type in Escherichia coli strains isolates from community-acquired urinary tract infections (CA-UTI) has been observed. However little is known on the prevalence and risk factors of this global threat in developing countries.

Objective: The aim of this study was to study the prevalence and risk factors for CA-UTI in Yaoundé, Cameroon.

Methods: Eighty six patients with urinary E.coli infection recruited from 10 health structures in the town of Yaoundé, Cameroon. After taking the first urine, faeces were collected from the patients for the study of the intestinal flora. The sample collection of faeces was done on a selected gel of enterobacteria resistant to third generation of cephalosporin. The molecular typing of extended-spectrum $\beta$-lactamase (ESBL) was carried out.

Results: Eighty-six strains of E. coli from 86 patients were included. We found that $39(45.3 \%)$ strains produced an extendedspectrum beta-lactamase. Among risk factors, previous use of antibiotic and the dry season were associated with the presence of an ESBL-producing strain in the urine. All ESBL were identified as CTX-M. The production of CTX-M was found to be significantly associated with resistance to fluoroquinolones, aminoglycosides and to the association of trimethoprim-sulfamethazole.

Conclusions: The prevalence of CTX-M ESBL in Yaoundé, Cameroon, provides new evidence on the global dissemination of CTX-M and the extent of this phenomenon in developing countries.
\end{abstract}

Key Words: Urinary tract infection, Community, Escherichia coli ESBL, CTX-M, Cameroon.

\section{INTRODUCTION}

Urinary tract infections (UTIs) are a major public health problem in terms of morbidity and financial cost. ${ }^{[1]}$ There are currently 150 million UTIs per year worldwide and this remains a major health problem in developing coun- tries. ${ }^{[2]}$ These infections are mostly caused by bacteria and acquired in community. ${ }^{[3]}$ Women are mostly affected. ${ }^{[4]}$ Escherichia coli is responsible for $75 \%-90 \%$ of all urinary tract infections in community patients. Many studies of antimicrobial susceptibility of $E$. coli strains in urinary commu-

\footnotetext{
*Correspondence: Ingrid Cécile Djuikoue; Email: djuikoe1983@yahoo.fr; Address: Unités de Santé Publique \& Epidémiologie, et de Microbiologie, Université des Montagnes, Bangangté, Cameroun.
} 
nity patients were performed in industrialized countries. ${ }^{[3]}$ The global spreading of CTX-M extended-spectrum betalactamase (ESBL) in these strains E.coli was observed and is been described as pandemic. ${ }^{[5]}$ There is paucity of reports on ESBL in E. coli strains in community-acquired UTIs in developing countries. ${ }^{[2,6-8]}$ Few studies have been done on ESBL however they only looked at intestinal carriage. ${ }^{\text {[9-11] }}$ The prevalence of CTX-M in E. coli strains responsible for community-acquired UTI in Cameroon or Central Africa has not been documented. The aim of our study was to determine the prevalence of bla CTX-M-type in E. coli strains in community-acquired urinary tract infections in women as well as its risk factors in Cameroon.

\section{MethodS}

From May 2011 to April 2012, we recruited all women who had UTI in 10 healthcare facilities (3 public hospitals and 7 laboratories in Yaoundé, Cameroon). Only those who had signed a consent form, filled and questionnaire on clinical data (history of UTIs, antibiotic use in the three preceding months, hospitalization in the last three months and the presence of burn) and demographical data and submitted a sample of stool were included. 86 smples from women with biological signs of $E$. coli UTI (leukocyturia $>10^{5}$ leukocytes per ml of urine) and $E$. coli bacteriuria ( $>10^{5}$ UFC per $\mathrm{ml}$ of urine) were isolated in Cytine Lactose Electrolyte Deficient and identified by the API 20 E gallery (bioMérieux, Marcy l'Etoile, France). E. coli strains were stored in $1 \mathrm{ml}$ of brain heart broth, supplemented with $10 \%$ glycerol and frozen at $-80^{\circ} \mathrm{C}$ until shipped together with the stool samples to the Bacteriology Laboratory of the Bichat-Claude Bernard
Hospital, Paris, France were analyses were done.

\subsection{Antimicrobial drug susceptibility and ESBL confir- matory testing}

E. coli urinary strains were reseeded on Trypticase Soja Agar (TSA) incubated at $37^{\circ} \mathrm{C}$ for 18 to 24 hours, and a reidentification was made using mass spectrometry, MALDITOF (Brucker, Bremen, Germany). ESBL phenotype was identified using E. coli urinary isolates by the Mueller-Hinton agar disc diffusion method as recommended by the Antibiogram Committee of the French Society for Microbiology (Comité d'antibiogramme de la société française de Microbiologie). Susceptibility to amoxicillin tircacillin, amoxicillin + clavulanic acid, cefotaxime, cefoxitin, ceftazidime, cefepime, ertapenem, nalidixic acid, ofloxacin, ciprofloxacin. gentamicin kanamycin, amikacin, fosfomycin, sulfamthoxazoletrimethoprim were determined using the disk-diffusion method (Bio-Rad, Marnes-la-Coquette, France). Results were interpreted according to the recommendations from the French Society for Microbiology (Comité d'antibiogramme de la société française de Microbiologie).

\subsection{Fecal antibiotic activity}

Exposition to antibiotics at the time of fecal sampling was defined by the detection of fecal antibiotic activity. Fecal antibiotic activity was detected using a simple microbiological assay, performed as described elsewhere. ${ }^{[12]}$

\subsection{PCR amplification}

This was done as decribed previously by Ruppé et al. ${ }^{[2]}$ except that we used 1 colony and $1.7 \%$ agar gel on Sybergreen (see Table 1).

Table 1. Primers used to study CTX-M $\beta$-lactamases in Escherichia coli, Cameroun, 2011-2012

\begin{tabular}{llll}
\hline Gene detected & Primer name & Primer sequence $(\mathbf{5}$ ' $\rightarrow \mathbf{3}$ ') & Reference \\
\hline${ }^{b l a}$ TEM & C & TCG GGG AAA TGT GCG CG & {$[13]$} \\
& D & TGC TTA ATC AGT GAG GCA CC & \\
${ }^{b l a}$ SHV & OS-5 & TTA TCT CCC TGT TAG CCA CC & {$[14]$} \\
& OS-6 & GAT TTG CTG ATT TCG CTC GG & \\
${ }^{b l a} C T X-M$ group 1 & M13U & GGT TAA AAA ATC ACT GCG TC & {$[13]$} \\
& M13L & TTG GTG ACG ATT TTA GCC GC & \\
\hline
\end{tabular}

\subsection{Statistical analysis}

We used for analysis the $\mathrm{R}$ software, version 3.1.0. Risk factors were identified using univariate analysis with the $\mathrm{Chi}^{2}$ or Fisher exact tests for qualitative variables of ANOVA for quantitative variables. All variables in the univariate analysis with a $P$-value less than .20 were introduced into a multivariate logistic model, using the backward strategy and testing some possible interactions. Some variables such as: the presence of antibiotics in stool and hospitalization in the past three months were systematically maintained in the model. Statistical differences with a $p$-value less than .05 were considered significant.

\subsection{Ethics considerations}

The Cameroon National Ethical Committee issued an ethical clearance (approval No. 207/CNE/SE/2011). After the main 
investigator had explained and read the project, all patients signed a consent form.

\section{Results}

\subsection{Population characteristics}

We recruited a total of 86 patients in 3 government hospitals and seven laboratories. The median age was 33 years (IQR 22.75). Twelve patients (14\%) had a history of urinary tract infection. The use of antibiotics at the time of sampling was found in about one third patients and $29.1 \%$ in the three months prior to inclusion. $12 \%$ of patients were hospitalized in the three months before the inclusion, and $11 \%$ of the patients had undergone surgery.

Eighty six E. coli strains were isolated. Among these, $74.4 \%$ of patients lived in the Center region, $11.6 \%$ were from the East, $10.5 \%$ from the West, $2.3 \%$ from the Littoral and $1.2 \%$ from the North West regions respectively.

\subsection{Prevalence of antimicrobial drug resistance in E. coli strains}

Almost half of the strains 39/86 (45.35\%) were producing ESBL CTX-M. These strains were co-resistant to quinolones (94.9\% to nalidixic acid), to fluoroquinolones (82.1\% to ciprofloxacin), to aminoglycosides (97.4\% to gentamicin, $87.2 \%$ to kanamycin and $30.8 \%$ to amikacin), $100 \%$ to trimethoprim-sulfamethazole and $7.7 \%$ to fosfomycin. No resistance to ertapenem and cefoxitin was observed.

\subsection{Beta-lactamase characterization}

All thirty nine strains of $E$. coli with an ESBL phenotype were analyzed. All ESBL producing E. coli strains were CTX-M of group 1. No strain of E.coli had TEM or SHV.

\subsection{Risk factors associated with ESBL $E$. coli urinary tract infection}

The mean age of patients with ESBL producing E. coli infection was 39.8 years against 36.7 years for patients with nonESBL producing E. coli strain infection.

The univariate analysis (see Table 2) showed that factors associated with the occurrence of urinary tract infections were the dry season, the presence of a burn and presence of antibiotic in stool. Age of patients, previous hospitalizations for cases such as diabetes and kidney failure were not significantly associated with the occurrence of ESBL urinary tract infections.

The multivariate analysis as presented in Table 3 showed that the dry season (OR $=17.13,95 \% \mathrm{CI}[3.1$ to 95.0$])$, the occurrence of a burn (OR $=5.4,95 \% \mathrm{CI}$ [1.6 to 18.1]) and the absence of urinary recurrence $(\mathrm{OR}=7.7,95 \%$ CI $[1.4$ to 43.1]) were independently associated with ESBL E. coli urinary tract infections. With a $p$ value at the limit of significance, patients who have consumed antibiotics in the last three months were more likely to be infected by ESBL $E$. coli strains $(\mathrm{OR}=4.4,95 \%$ CI [1.1-16.5]).

Table 2. Univariate analysis of associated risk factors

\begin{tabular}{|c|c|c|c|c|}
\hline & \multicolumn{2}{|c|}{ Presence of ESBL } & \multirow[t]{2}{*}{ OR.95.CI. } & \multirow[t]{2}{*}{$P$} \\
\hline & Yes & No & & \\
\hline Total & $39(45.4)$ & 47 (54.6) & & \\
\hline \multicolumn{5}{|l|}{ Season of the year } \\
\hline Rainy & $2(5.1)$ & $22(46.8)$ & Ref & \\
\hline Dry & 37 (94.9) & $25(53.2)$ & $16.28(3.51,75.48)$ & $<.001$ \\
\hline Patient's home region & & & & .382 \\
\hline Centre & $30(76.9)$ & $34(72.3)$ & Ref & \\
\hline East & $4(10.3)$ & $6(12.8)$ & $0.76(0.19,2.94)$ & .686 \\
\hline Littoral & $0(0)$ & $2(4.3)$ & $0(0$, Inf $)$ & .992 \\
\hline Northwest & $0(0)$ & $1(2.1)$ & $0(0$, Inf $)$ & .995 \\
\hline West & $5(12.8)$ & $4(8.5)$ & $1.42(0.35,5.76)$ & .627 \\
\hline \multicolumn{5}{|l|}{ Age } \\
\hline median(IQR) & $37(26,49.5)$ & $32(23,46.5)$ & $\begin{array}{l}1.0076 \\
(0.9867,1.029)\end{array}$ & .479 \\
\hline \multicolumn{5}{|l|}{$\begin{array}{l}\text { Hospital } \\
\text { training/Laboratory }\end{array}$} \\
\hline Public & 19 (48.7) & $27(57.4)$ & Ref & \\
\hline Private & $20(51.3)$ & $20(42.6)$ & $1.42(0.61,3.34)$ & .42 \\
\hline \multicolumn{5}{|l|}{ Burn } \\
\hline Yes & $22(56.4)$ & $14(29.8)$ & Ref & \\
\hline No & 17 (43.6) & $33(70.2)$ & $0.33(0.13,0.8)$ & .014 \\
\hline \multicolumn{5}{|l|}{ Lumbar pain } \\
\hline Yes & $9(23.1)$ & $14(29.8)$ & Ref & \\
\hline No & $30(76.9)$ & $33(70.2)$ & $\begin{array}{l}1.41 \\
(0.53,3.74)\end{array}$ & .485 \\
\hline $\begin{array}{l}\text { Antibiotics at the time } \\
\text { of sampling }\end{array}$ & & & & .948 \\
\hline Yes & $12(30.8)$ & $13(27.7)$ & Ref & \\
\hline No & $14(35.9)$ & $18(38.3)$ & $\begin{array}{l}0.84 \\
(0.29,2.41)\end{array}$ & .749 \\
\hline Do not know & $13(33.3)$ & $16(34)$ & $0.88(0.3,2.57)$ & .816 \\
\hline \multicolumn{5}{|l|}{$\begin{array}{l}\text { History of urinary } \\
\text { tract infections }\end{array}$} \\
\hline Yes & $3(7.7)$ & $8(17)$ & Ref & \\
\hline No & $36(92.3)$ & $39(83)$ & $2.46(0.61,10)$ & .208 \\
\hline \multicolumn{5}{|l|}{$\begin{array}{l}\text { Recurrences of urinary } \\
\text { tract infections }\end{array}$} \\
\hline Yes & $3(7.7)$ & $9(19.1)$ & Ref & \\
\hline No & $36(92.3)$ & 38 (80.9) & $\begin{array}{l}2.84 \\
(0.71,11.34)\end{array}$ & .139 \\
\hline $\begin{array}{l}\text { Risk factors for } \\
\text { urinary tract infections }\end{array}$ & & & & \\
\hline Yes & $23(59)$ & 23 (48.9) & Ref & \\
\hline No & $16(41)$ & $24(51.1)$ & $0.67(0.28,1.57)$ & 0.354 \\
\hline \multicolumn{5}{|l|}{ Urological history } \\
\hline Yes & $0(0)$ & $2(4.3)$ & Ref & \\
\hline No & 39 (100) & 45 (95.7) & $13564512.33(0$, Inf $)$ & 0.992 \\
\hline
\end{tabular}


Table 2. (continued.)

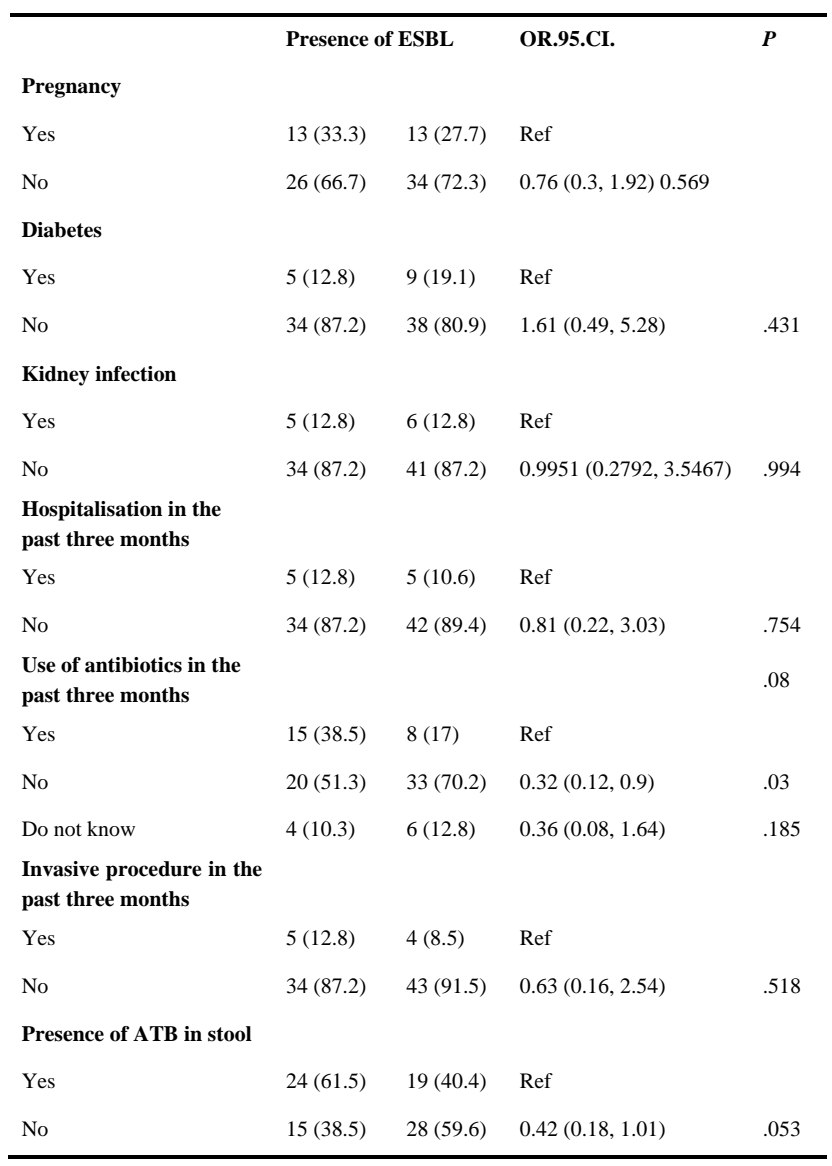

Table 3. Multivariate analysis

\begin{tabular}{lll}
\hline Variables & OR.95.CI. & P-value \\
\hline $\begin{array}{l}\text { Season of the year } \\
\text { Rainy }\end{array}$ & Ref & \\
Dry & $17.13(3.09,94.99)$ & .001 \\
Burn & & \\
No & Ref & \\
Yes & $5.45(1.64,18.08)$ & .006 \\
Recurrences of urinary tract infections & & \\
Yes & Ref & .02 \\
No & $7.73(1.39,43.08)$ & .072 \\
Use of antibiotics in the past three months & & \\
No & Ref & .03 \\
Yes & $4.36(1.15,16.49)$ & .742 \\
Do not know & $1.35(0.22,8.21)$ & \\
Presence of ATB in stool & & .114 \\
\hline No & Ref & \\
Yes & $2.46(0.81,7.53)$ & \\
\hline
\end{tabular}

\section{Discussion}

Our study is the first study on E. coli producing CTX -M in UTI among women in Cameroon. Although the number of acquired strains may be considered to be limited $(n=86)$, their level of antibiotic resistance is quite high regarding their community origin. As such, 39 strains of $E$. coli of community origin had an ESBL CTX-M.
Our study was conducted on the same target population and followed a similar protocol as those used in Senegal. The average prevalence found in this country was $6.5 \% .^{[15]}$ Meanwhile the prevalence found in Cameroon was $45.3 \%$. This result is higher than the Senegal study carried out 10 years ago.

In Cameroon, all E. coli strains isolated CTX-M are coresistant to other antibiotics families such as aminoglycosides, fluoroquinolones, trimethoprimsulfamethazole. A study in Dakar revealed low resistance rates of Enterobacteriaceae to tromethanolfosfomycin, a form of fosfomycin characterized by a high oral bioavailability. ${ }^{[16]}$ Findings obtained in that study corroborates our results. Fosfomycin may be recommended as a reliable empirical treatment for simple urinary tract infections because of its ease in use (one dose), good tolerance and effectiveness. ${ }^{[17]}$

The high prevalence of antibiotic resistance in the community would also be associated to the poor hygiene in Cameroon. Since it is densely populated. During the dry season access to drinking water is a major problem. Water certainly promotes the circulation of Enterobacteriaceae strains (be they resistant or not). ${ }^{[18-25]}$ Moreover, access to drinking water is not properly insured in many parts of the city. As such, Enterobacteriaceae from stools can easily affect many people through contaminated water. Many households get water from unhealthy streams used as a dumping ground by residents. A study carried out by De Boeck in Kinshasa (Central Africa ) revealed the presence of ESBL producing Enterobacteriaceae in drinking water ${ }^{[26]}$ and another study revealed the presence of $E$. coli in rivers and wells. ${ }^{[21]}$ In a village community in South Guyana, it was also proven that even in the absence of a strong antibiotic pressure, promiscuity promoted the circulation of resistant strains and thus increased the prevalence of carrying them. ${ }^{[27]}$ The uncontrolled consumption of antibiotics may promote the selection of E. coli strains producing ESBL, which usually results in the empirical prescription for self-medication, poor adherence of patients to antibiotic therapy, the quality of antibiotics (generic under dosed) and the questionable preservation (street drugs). The prescription of antibiotics is in most cases dispensed without microbiological data, and is therefore based on clinical and also on the patient's ability to pay his/her antibiotics. This also applies to medical tests, which are non-refundable, and this is because Cameroon has not implemented the social security system.

In addition, most of these strains were co-resistant to fluoroquinolones, aminoglycosides and trimethoprimsulfamethazole, thus drastically reducing therapeutic options in the treatment of such infections. 


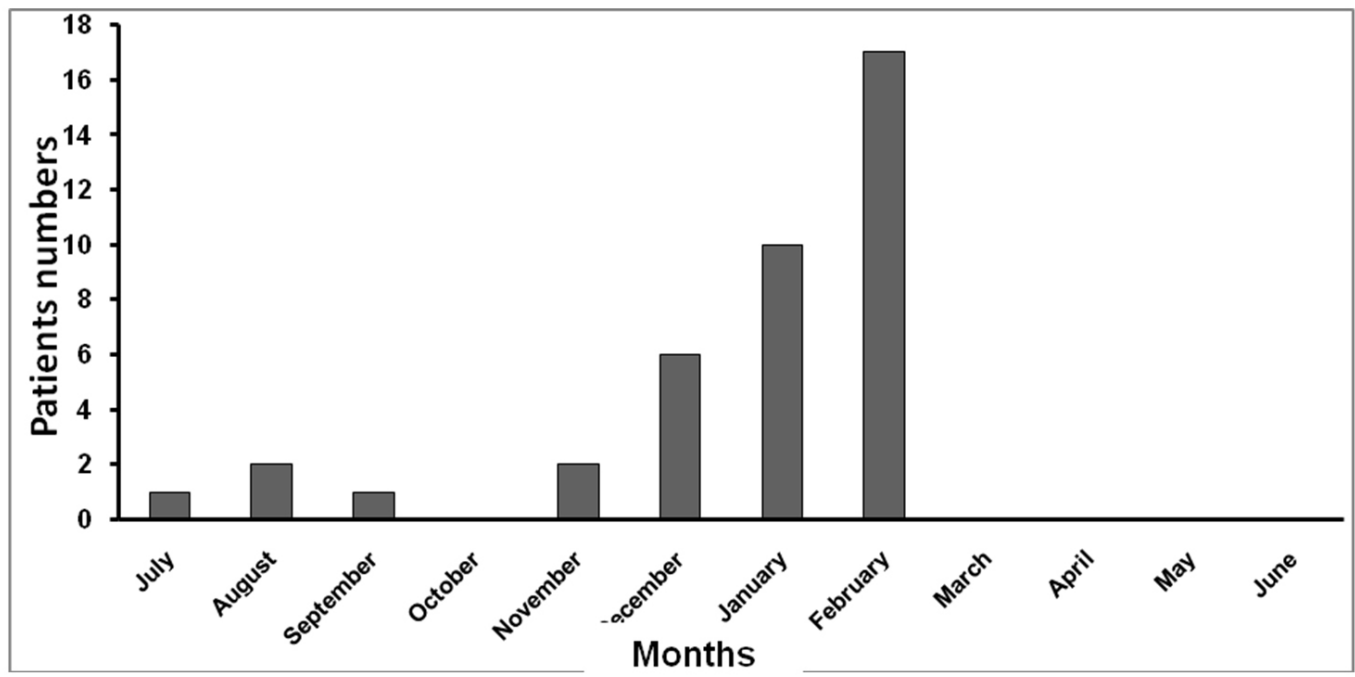

Figure 1. Number of ESBL E. coli cases according to the month of collection

\section{Conclusion}

The findings of this study show that the global dissemination of CTX-M-type ESBL affects particularly Cameroon and its capital city, since nearly half of the community strains of E. coli accounting for urinary tract infections had this type of ESBL. Furthermore, these strains were in most cases coresistant to other antibiotics widely used in E. coli infection treatments.

The situation of CTX-M in Cameroon he brings new facts to explain the current global pandemic. Hygiene and the normal use of antibiotics must be in the centre for the fight against the spread of such multidrug-resistant strains.

We believe it is right time authorities in Africa in general and Cameroon in particular should be concerned by providing health facilities structures to carry out complete bacteriological analysis, harmonize antibiogram procedures in order to detect bacteria producing extended spectrum betalactamases and to stop the use of antibiotics such as amoxicillin, ciprofloxacin and cotrimozaxole in empirical treat- ment since there's a strong resistance of Enterobacteriaceae to these antibiotics. Fosfomycin may be recommended as a reliable empirical treatment for urinary tract infections, unfortunately this antibiotic is not yet available in Cameroon.

\section{ACKNOWLEDGeMENTS}

This work was partly supported by the Agence Universitaire de la Francophonie (AUF), the Bacteriological Laboratory of the University Training Hospital (CHU) Bichat Paris and The National Reference Centre for Bacterial Resistance in Commensal Flora. The authors wish to thank Dr. Aghokeng Avelin, Dr. Fosso, Dr Tchattad, Dr. Nkoa, Dr. Kouinche Adelaide, Dr. Fokam Joseph, Ms. Djoko Djuikouo Bibiane AICHA, Ms Mawane Leonie,Mr Pouma, Mr. DJAMARDIN Ms. Colette Ngono and Mr. Jean-Bapiste Ketchiewouo for their contribution to patient sampling process and for the preservation of strains.

\section{CONFlicts of InTEREST Disclosure}

Authors declare that they have no competing interests.

\section{REFERENCES}

[1] Flores-Mireles, Ana L, Walker JN, et al. Urinary Tract Infections: Epidemiology, Mechanisms of Infection and Treatment Options. Nature Reviews. Microbiology. 2015; 13(5): 269-84.

[2] Ruppé E, Hem S, Lath S, et al. CTX-M Beta-Lactamases in Escherichia Coli from Community-Acquired Urinary Tract Infections, Cambodia. Emerging Infectious Diseases. 2009; 15(5): 741-48. PMid:19402960 https://doi.org/10.3201/eid1505.071299

[3] Hertz FB, Nielsen JB, Schønning K, et al. Population Structure of Drug-Susceptible-resistant and ESBL-Producing Escherichia Coli from Community-Acquired Urinary Tract. BMC Microbiology. 2016;

Published by Sciedu Press
16(1): 1-1.

[4] Schappert SM, Rechtsteiner EA. Ambulatory Medical Care Utilization Estimates for 2007. Vital Health Stat 13. 2011; 169: 1-38.

[5] Cantón R, Coque TM. The CTX-M Beta-Lactamase Pandemic. Current Opinion in Microbiology. 2006; 9(5): 466-75. https: //doi.org/10.1016/j.mib.2006.08.011

[6] Sire JM, Nabeth P, Perrier-Gros-Claude JD, et al. Antimicrobial Resistance in Outpatient Escherichia Coli Urinary Isolates in Dakar, Senegal. The Journal of Infection in Developing Countries. 2007; 1(03): 263-68.

[7] Woerther PL, Angebault C, Jacquier H, et al. Massive Increase, 
Spread, and Exchange of Extended Spectrum $\beta$-Lactamase-Encoding Genes among Intestinal Enterobacteriaceae in Hospitalized Children with Severe Acute Malnutrition in Niger. Clinical Infectious Diseases: An Official Publication of the Infectious Diseases Society of America. 2011; 53(7): 677-85. https://doi.org/10.1093/cid/cir522

[8] Kiiru J, Kariuki S, Goddeeris BM, et al. Analysis of $\beta$-Lactamase Phenotypes and Carriage of Selected $\beta$-Lactamase Genes among Escherichia Coli Strains Obtained from Kenyan Patients during an 18-Year Period. BMC Microbiology. 2012; 12: 155. https: //doi.org/10.1186/1471-2180-12-155

[9] Gangoué-Piéboji J, Bedenic B, Koulla-Shiro S, et al. Extendedspectrum-beta-lactamase-producing Enterobacteriaceae in Yaounde, Cameroon. J Clin Microbiol. 2005; 43(7): 3273-7.

[10] Lonchel CM, Meex C, Gangoué-Piéboji J, et al. Proportion of extended-spectrum B-lactamase-producing Enterobacteriaceae in community setting in Ngaoundere, Cameroon. BMC Infect Dis. 2012; 9; 12: 53. https://doi.org/10.1186/1471-2334-12-53

[11] Djuikoue IC, Woerther PL, Toukam M, et al. Intestinal carriage of Extended Spectrum Beta-Lactamase producing E. coli in women with urinary tract infections, Cameroon. J Infect Dev Ctries. 2016; 10(10): 1135-1139. https://doi.org/10.3855/jidc. 7616

[12] Messer JW, Leslie JE, Houghtby GA, et al. Bacillus Stearothermophilus Disc Assay for Detection of Inhibitors in Milk: Collaborative Study. Journal-Association of Official Analytical Chemists. 1982; 65(5): 1208-14.

[13] Saladin M, Cao VT, Lambert T, et al. Diversity of CTX-M BetaLactamases and Their Promoter Regions from Enterobacteriaceae Isolated in Three Parisian Hospitals. FEMS Microbiology Letters. 2002; 209(2): 161-68.

[14] Arlet G, Rouveau M, Philippon A. Substitution of Alanine for Aspartate at Position 179 in the SHV-6 Extended-Spectrum BetaLactamase. FEMS Microbiology Letters. 1997; 152(1): 163-67. https ://doi.org/10.1016/S0378-1097(97)00196-1

[15] Dromigny JA, Nabeth P, Juergens-Behr A, et al. Risk Factors for Antibiotic-Resistant Escherichia Coli Isolated from CommunityAcquired Urinary Tract Infections in Dakar, Senegal. The Journal of Antimicrobial Chemotherapy. 2005; 56(1): 236-39. https: //doi.org/10.1093/jac/dki158

[16] Nabeth P, Perrier-Gros-Claude JD, Juergens-Behr A, et al. In Vitro Susceptibility of Quinolone-Resistant Enterobacteriaceae Uropathogens to Fosfomycin Trometamol, in Dakar, Senegal. Scandinavian Journal of Infectious Diseases. 2005; 37(6-7): 497-99. https://doi.org/10.1080/00365540510038956

[17] Lobel B. Short Term Therapy for Uncomplicated Urinary Tract Infection Today. Clinical Outcome Upholds the Theories. International Journal of Antimicrobial Agents. 2003; 22(Supplement 2): 85-87. https://doi.org/10.1016/S0924-8579(03)00237-1
[18] Dolejska M, Frolkova P, Florek M, et al. CTX-M-15-Producing Escherichia Coli Clone B2-O25b-ST131 and Klebsiella Spp. Isolates in Municipal Wastewater Treatment Plant Effluents. The Journal of Antimicrobial Chemotherapy. 2011; 66(12): 2784-90. https: //doi.org/10.1093/jac/dkr363

[19] Reinthaler FF, Feierl G, Galler H, et al. ESBL-Producing E. Coli in Austrian Sewage Sludge. Water Research. 2010; 44(6): 1981-85. https://doi.org/10.1016/j.watres.2009.11.052

[20] Chagas TPG, Seki LM, Cury JC, et al. Multiresistance, BetaLactamase-Encoding Genes and Bacterial Diversity in Hospital Wastewater in Rio de Janeiro, Brazil. Journal of Applied Microbiology. 2011; 111(3): 572-81. https ://doi.org/10.1111/j.1365 $-2672.2011 .05072 . \mathrm{x}$

[21] Amaya E, Reyes D, Paniagua M, et al. Antibiotic Resistance Patterns of Escherichia Coli Isolates from Different Aquatic Environmental Sources in León, Nicaragua. Clinical Microbiology and Infection: The Official Publication of the European Society of Clinical Microbiology and Infectious Diseases. 2012; 18(9): E347-354. https : //doi .org/10.1111/j.1469-0691.2012.03930.x

[22] Zurfluh K, Hächler H, Nüesch-Inderbinen M, et al. Characteristics of Extended-Spectrum $\beta$-Lactamase- and Carbapenemase-Producing Enterobacteriaceae Isolates from Rivers and Lakes in Switzerland. Applied and Environmental Microbiology. 2013; 79(9): 3021-26. https://doi.org/10.1128/AEM.00054-13

[23] Kim J, Kang HY, Lee Y. The Identification of CTX-M-14, TEM52, and CMY-1 Enzymes in Escherichia Coli Isolated from the Han River in Korea. Journal of Microbiology (Seoul, Korea). 2008; 46 (5): 478-81. https://doi.org/10.1007/s12275-008-0150-y

[24] Alouache S, Kada M, Messai Y, et al. Antibiotic Resistance and Extended-Spectrum $\beta$-Lactamases in Isolated Bacteria from Seawater of Algiers Beaches (Algeria). Microbes and Environments/JSME. 2012; 27(1): 80-86. https ://doi .org/10.1264/jsme2.ME1126 6

[25] De Boeck H, Lunguya O, Muyembe JJ, et al. Presence of ExtendedSpectrum Beta-Lactamase-Producing Enterobacteriaceae in Waste Waters, Kinshasa, the Democratic Republic of the Congo. European Journal of Clinical Microbiology \& Infectious Diseases: Official Publication of the European Society of Clinical Microbiology. 2012; 31(11): 3085-88. https://doi.org/10.1007/s10096-0 12-1669-8

[26] De Boeck H, Miwanda B, Lunguya-Metila O, et al. ESBL-Positive Enterobacteria Isolates in Drinking Water. Emerging Infectious Diseases. 2012; 18(6): 1019-20. https://doi.org/10.3201/eid1 806.111214

[27] Grenet K, Guillemot D, Jarlier V, et al. Antibacterial Resistance, Wayampis Amerindians, French Guyana. Emerging Infectious Diseases. 2004; 10(6): 1150-53. https://doi.org/10.3201/eid1 006.031015 